\title{
Adaptive Time-Splitting Scheme for Nanoparticles Transport with Two-phase Flow in Heterogeneous Porous Media
}

\author{
Mohamed F. El-Amin ${ }^{\mathrm{a}, \mathrm{b}, \mathrm{c}, \mathrm{e}}$, Jisheng Kou ${ }^{\mathrm{d}}$, Shuyu Sun ${ }^{\mathrm{c}}$ \\ ${ }^{a}$ Effat University, Jeddah 21478, Kingdom of Saudi Arabia. \\ ${ }^{b}$ Mathematics Department, Faculty of Science, Aswan University, Aswan 81528, Egypt. \\ ${ }^{c}$ Computational Transport Phenomena Laboratory (CTPL), Division of Physical Sciences \\ and Engineering (PSE), King Abdullah University of Science and Technology (KAUST), \\ Thuwal, Kingdom of Saudi Arabia. \\ ${ }^{d}$ School of Mathematics and Statistics, Hubei Engineering University, Xiaogan 432000, \\ Hubei, China. \\ ${ }^{e}$ momousa@effatuniversity.edu.sa
}

\begin{abstract}
In this work, we introduce an efficient scheme using an adaptive time-splitting method to simulate the problem of nanoparticles transport with a two-phase flow in heterogeneous porous media. The pressure and saturation equations are coupled with the capillary pressure which is linearized in terms of saturation. An IMplicit Pressure Explicit Saturation-IMplicit Concentration (IMPES-IMC) scheme is used to solve the problem under consideration. The cell-centered finite difference (CCFD) method is used for the spatial discretization. The external time interval is divided into three levels, the first level is for the pressure, the second level of the saturation, while the third level is applied for the concentrations. This method can reduce the computational cost arisen from the implicit solution of the pressure equation and concentration equation as well as the rapid changes in saturation and concentration.
\end{abstract}


The time step-sizes for saturation and concentration equations are adaptive under computing and satisfying the Courant-Friedrichs-Lewy $\left(\mathrm{CFL}_{\mathbf{j}} 1\right)$ condition, iteratively. These results show the good performance of the scheme. Moreover, a numerical example of a highly heterogeneous porous medium is introduced and adaptive time step-sizes are shown in graphs for different cases, in addition to saturation, nanoparticles concentration contours.

Keywords: time-splitting, IMPES, nanoparticles, two-phase flow, porous media, reservoir simulation

\section{Introduction}

The IMplicit Pressure Explicit Saturation (IMPES), is a conditionally stable approach, solves the pressure equation implicitly and updates the saturation explicitly. Hence it takes a very small time step size, in particular with heterogeneous porous media. The IMPES scheme has been improved in several versions (e.g. $[1,2,3,4,5])$. The temporal discretization scheme is considered an important factor that affects the efficiency of numerical reservoir simulators. The application of traditional single-scale temporal scheme is restricted by the rapid changes of the pressure and saturation with capillarity and concentrations if applicable. So, applying time splitting strategies has a significant improvement to treating the gap between the pressure and the saturation. Time splitting method has been considered in a number of publications such as [6]-[15]. For example, in Refs. [11, 12], an explicit subtiming scheme is provided. On the other hand, an implicit time-stepping 
scheme have been proposed by Bhallamudi et al. [9]. Similar methodology has been used to the problem of flow and transport simulations in fractured porous media [10]. VanderKwaak [16] have introduced a hybrid implicitexplicit scheme by treating implicitly some portions of the domain under certain stability conditions, while the rest of the domain is treated by the explicit time-stepping approach.

Recently, applications of nanoparticles have been considered in many branches of petroleum engineering, especially, enhanced oil recovery. Ju et al. $[19,20]$ have formulated a mathematical model of nanoparticles transport in two-phase flow in porous media based on the work in Refs. [21]. El-Amin et al. $[22,23]$ have introduced a model to simulate nanoparticles transport in two-phase flow in porous media. In [24], authors extended the model to include a negative capillary pressure and mixed relative permeabilities correlations to fit with the mixed-wet system. In Ref. [25], they have introduced numerical and dimensional analyses of nanoparticles transport with a twophase flow in porous media. Salama et al. [26] have investigated the problem of transport of nanoparticles in anisotropic porous media numerically using the multipoint flux approximation. Recently, Chen et al. [27] presented a numerical simulation of drag reduction effects by hydrophobic nanoparticles adsorption method in water flooding processes. Chen et al. [28] have considered the dynamic update of anisotropic permeability field during the transport of nanoparticles in the subsurface. In [29] we have introduced nonlinear iterative IMPES-IMC (IMplicit Pressure Explicit Saturation-IMplicit Con- 
centration) scheme to solve the flow equation of the model of nanoparticles transport in porous media.

In the current work, we introduce a time-stepping technique for the modeling and simulation of nanoparticles transport with two-phase flow. An IMPES-IMC scheme is used to solve the problem under consideration, while the CCFD method is used for the spatial discretization. The time-stepping scheme is applied under the CFL condition to adapting the time-steps sizes. Finally, numerical experiments are provided. The paper is organized as follows: The second section is devoted to the modeling and mathematical formulation. The third section is devoted to the time-stepping technique. The time-steps adaption is provided in the fourth section. The CCFD spatial discretization is introduced in Sec. 5. Then, Sec. 6 is devoted to numerical tests, while the conclusions are presented in Sec. 7 .

\section{Modeling and Mathematical Formulation}

This paper considers the problem of nanoparticles transport with a twophase immiscible incompressible flow in porous media, the system of governing equations consists of water saturation, Darcy's law, nanoparticles concentration, deposited nanoparticles concentration on the pore-wall, and entrapped nanoparticles concentration in the pore-throat. Also, the porosity and permeability vary due to the nanoparticles deposition/entrapment on/in the pores. In the following we introduce the governing equations briefly, (for details see Refs. [22, 23, 24, 25, 26, 28, 29]: 
Momentum Conservation (Darcy's Law):

$$
\mathbf{u}_{\alpha}=-\frac{k_{\alpha}}{\mu_{\alpha}} \nabla \Phi_{\alpha}, \quad \alpha=w, n
$$

where

$$
k_{\alpha}=k_{r \alpha} \mathbf{K}, \quad \Phi_{\alpha}=p_{\alpha}+\rho_{\alpha} g \nabla z, \quad \alpha=w, n,
$$

\section{Mass Conservation (Saturation Equations):}

$$
\phi \frac{\partial s_{\alpha}}{\partial t}+\nabla \cdot \mathbf{u}_{\alpha}=q_{\alpha}, \quad \alpha=w, n
$$

where

$$
s_{w}+s_{n}=1 \text {. }
$$

where $\mathbf{K}$ is the permeability tensor $\mathbf{K}=k \mathbf{I}$, where $\mathbf{I}$ is the identity matrix and $k$ is a positive real number. $\phi$ is the porosity, $g$ is the gravitational acceleration, and $z$ is the depth. $\mathbf{u}_{\alpha}, \Phi_{\alpha}, p_{\alpha}, \mu_{\alpha}, \rho_{\alpha}, k_{\alpha}, k_{r \alpha}, q_{\alpha}, s_{\alpha}$ are, respectively, the velocity, the pressure potential, the pressure, the viscosity, the density, the effective permeability, the relative permeability, the external mass flow rate, and the saturation of the phase $\alpha . w$ stands for the wetting phase (water), and $n$ stands for the nonwetting phase (oil).

Providing the following definitions:

The capillary pressure: $p_{c}\left(s_{w}\right)=p_{n}-p_{w}$.

The total velocity: $\mathbf{u}_{t}=\mathbf{u}_{w}+\mathbf{u}_{n}$. 
The flow fraction: $f_{w}=\lambda_{w} / \lambda_{t}$.

The phase mobility: $\lambda_{\alpha}=k_{r \alpha} / \mu_{\alpha}$.

The total mobility: $\lambda_{t}$.

The capillary pressure potential: $\Phi_{c}=p_{c}+\left(\rho_{n}-\rho_{w}\right) g \nabla z$.

The total source mass transfer: $q_{t}=q_{w}+q_{n}$.

After some mathematical manipulations and referring to Refs. [17], the pressure equation can be rewritten as,

$$
\nabla \cdot \mathbf{u}_{t}=-\nabla \cdot \lambda_{t} \mathbf{K} \nabla \Phi_{w}-\nabla \cdot \lambda_{n} \mathbf{K} \nabla \Phi_{c}=q_{t}
$$

Because this equation contents the capillary pressure which is a function of saturation, it will be coupled with the following saturation equation to calculate the pressure,

$$
\phi \frac{\partial s_{w}}{\partial t}-q_{w}=-\nabla \cdot \lambda_{w} \mathbf{K} \nabla \Phi_{w}
$$

However, the saturation is updated using the following form,

$$
\phi \frac{\partial s_{w}}{\partial t}-q_{w}=-\nabla \cdot\left(f_{w} \mathbf{u}_{a}\right)
$$

where $\mathbf{u}_{w}=f_{w} \mathbf{u}_{a}$ and $\mathbf{u}_{a}=-\lambda_{t} \mathbf{K} \nabla \Phi_{w}$.

\section{Nanoparticles Transport Model:}

Assuming that the nanoparticles exist only in the water phase of one size interval. So, The transport equation of the nanoparticles in the water phase 
is given as $([18,21,19,20,22,23,24,25,26,28,29])$,

$$
\phi \frac{\partial\left(s_{w} c\right)}{\partial t}+\nabla \cdot\left(\mathbf{u}_{w} c-D \nabla c\right)=R+Q_{c}
$$

\section{Nanoparticles Surface Deposition:}

The surface deposition is expressed by,

$$
\frac{\partial c_{s 1}}{\partial t}=\left\{\begin{array}{cc}
\gamma_{d}\left|\mathbf{u}_{w}\right| c, & \mathbf{u}_{w} \leq u_{r} \\
\gamma_{d}\left|\mathbf{u}_{w}\right| c-\gamma_{e}\left|\mathbf{u}_{w}-u_{r}\right| c_{s 1}, & \mathbf{u}_{w}>u_{r}
\end{array}\right.
$$

\section{Nanoparticles Throat Entrapment:}

The rate of entrapment of the nanoparticles is,

$$
\frac{\partial c_{s 2}}{\partial t}=\gamma_{p t}\left|\mathbf{u}_{w}\right| c
$$

where $c$ is the nanoparticles concentrations. $\tau$ is the tortuosity parameter, of the flow of the water phase. $Q_{c}$ is the rate of change of particle volume belonging to a source/sink term. $c_{s 1}$ is the deposited nanoparticles concentration on the pore surfaces of the porous medium. $c_{s 2}$ is the entrapped nanoparticles concentration in pore throats. $\gamma_{d}$ is the rate coefficients for surface retention of the nanoparticles. $\gamma_{e}$ is the rate coefficients for entrainment of the nanoparticles. $u_{r}$ is the critical velocity of the water phase. where 
$\gamma_{p t}$ is the pore throat blocking constants. The diffusion-dispersion tensor is defined by,

$$
D=\phi s_{w} \tau D_{t}, \quad D_{t}=D^{\mathrm{Br}}+D^{\mathrm{disp}}
$$

where $B r$ is the Brownian diffusion and $D^{\text {disp }}$ is the dispersion coefficient which is defined by [2],

$$
\phi s_{w} \tau D^{\text {disp }}=d_{t, w}\left|\mathbf{u}_{w}\right| \mathbf{I}+\left(d_{l, w}-d_{t, w}\right) \frac{\mathbf{u}_{w} \mathbf{u}_{w}^{T}}{\left|\mathbf{u}_{w}\right|}
$$

Thus,

$$
D=\left(\phi s_{w} \tau D^{\mathrm{Br}}+d_{t, w}\left|\mathbf{u}_{w}\right|\right) \mathbf{I}+\left(d_{l, w}-d_{t, w}\right) \frac{\mathbf{u}_{w} \mathbf{u}_{w}^{T}}{\left|\mathbf{u}_{w}\right|}
$$

where $d_{l, w}$ and $d_{t, w}$ are the longitudinal and transverse dispersion coefficients, respectively. $R$ is the net rate of loss of nanoparticles which is defined by,

$$
R=\frac{\partial c_{s 1}}{\partial t}+\frac{\partial c_{s 2}}{\partial t}
$$

\section{Initial and Boundary Conditions:}

Consider the computational domain $\Omega$ with the boundary $\partial \Omega$ which is subjected to Dirichlet $\Gamma_{D}$ and Neumann $\Gamma_{N}$ boundaries, where $\partial \Omega=\Gamma_{D} \cup \Gamma_{N}$ and $\Gamma_{D} \cap \Gamma_{N}=\varnothing$. At the beginning of the injection process, we have,

$$
s_{w}=s_{w}^{0}, \quad c=c_{s 1}=c_{s 2}=0 \quad \text { in } \quad \Omega \quad \text { at } \quad t=0,
$$


The boundary conditions are given as,

$$
\begin{gathered}
p_{w}\left(\text { or } p_{n}\right)=p^{D} \quad \text { on } \quad \Gamma_{D}, \\
\mathbf{u}_{t} \cdot \mathbf{n}=q^{N}, \quad s_{w}=S^{N}, \quad c=c_{0}, \quad c_{s 1}=c_{s 2}=0 \quad \text { on } \quad \Gamma_{N} .
\end{gathered}
$$

where $\mathbf{n}$ is the outward unit normal vector to $\partial \Omega, p^{D}$ is the pressure on $\Gamma_{D}$ and $q^{N}$ the imposed inflow rate on $\Gamma_{N}$, respectively.

\section{Multi-scale Time Splitting Method}

The concept of time splitting method is to use different time step size for each equation has a time derivative. In the above-described method, the pressure is coupled with the saturation in each time-step. The timestep size for the pressure can be taken larger than the those of saturation and nanoparticle concentrations. So, for the pressure the total time interval $[0, T]$ is divided into $N_{p}$, time-steps as $0=t_{0}<t_{1}<\cdots<t_{N_{p}=T}$. Thus, the time-step length assigned for pressure is, $\Delta t^{k}=t^{k+1}-t^{k}$. Since the saturation varies more rapidly than the pressure, we use a smaller time-step size for the saturation equation. That is, each interval, $\left(t^{k}, t^{k+1}\right]$, will be divided into $N_{p, s}$ subintervals as $\left(t^{k}, t^{k+1}\right]=\cup_{l=0}^{N_{p, s}-1}\left(t^{k, l}, t^{k, l+1}\right]$. On the other hand, as the concentration varies more rapidly than the pressure (and may be saturation), we also use a smaller time-step size for the concentration equations. Thus, we partition each subinterval $\left(t^{k, l}, t^{k, l+1}\right]$ into $N_{p, s, c}$ sub-

subintervals as $\left(t^{k, l}, t^{k, l+1}\right]=\cup_{m=0}^{N_{p, s, c}-1}\left(t^{k, l, m}, t^{k, l, m+1}\right]$. Therefore, the system 
of governing equations, (3), (4), (6), (7) and (8), is solved based on the adaptive time-splitting technique. The backward Euler time discretization is used for the equations of pressure, saturation, concentration and the two volume concentration. We linearized the capillary pressure function, $\Phi_{c}$, in terms of saturation using this formula,

$$
\Phi_{c}\left(s_{w}^{*}\right) \cong \Phi_{c}\left(s_{w}^{k}\right)+\Phi_{c}^{\prime}\left(s_{w}^{k}\right)\left[s_{w}^{k+1}-s_{w}^{k}\right]
$$

where $\Phi_{c}^{\prime}$ is derivative of $\Phi_{c}$. The quantity, $\left[s_{w}^{k+1}-s_{w}^{k}\right]$, can be calculated from the saturation equation,

$$
s_{w}^{k+1}-s_{w}^{k}=\frac{\Delta t^{k}}{\phi\left(c_{s 1}^{k}, c_{s 2}^{k}\right)}\left[q_{w}^{k+1}-\nabla \cdot \lambda_{t}\left(s_{w}^{k}\right) \mathbf{K}\left(c_{s 1}^{k}, c_{s 2}^{k}\right) \nabla \Phi_{w}^{k+1}\right] .
$$

In addition to the pressure equation,

$$
-\nabla \cdot \lambda_{t}\left(s_{w}^{k}\right) \mathbf{K}\left(c_{s 1}^{k}, c_{s 2}^{k}\right) \nabla \Phi_{w}^{k+1}-\nabla \cdot \lambda_{n}\left(s_{w}^{k}\right) \mathbf{K}\left(c_{s 1}^{k}, c_{s 2}^{k}\right) \nabla \Phi_{c}\left(s_{w}^{*}\right)=q_{t}^{k+1}
$$

Then, the above coupled system (16), (17) and (18) is solved implicitly to obtain the pressure potential. Therefore, the saturation is updated explicitly with using the upwind scheme for the convection term as,

$$
\phi\left(c_{s 1}^{k}, c_{s 2}^{k}\right) \frac{s_{w}^{k, l+1}-s_{w}^{k, l}}{\Delta t^{l}}+\nabla \cdot\left(f_{w}^{k} \mathbf{u}_{a}^{k+1}\right)=q_{w}^{k, l+1}
$$


Therefore, the nanoparticles concentration, deposited nanoparticles concentration on the pore-walls and entrapped nanoparticles concentration in the pore-throats are computed implicitly as follow,

$$
\begin{aligned}
& \phi\left(c_{s 1}^{k}, c_{s 2}^{k}\right) \frac{s_{w}^{k+1} c^{k, l, m+1}-s_{w}^{k} c^{k, l, m}}{\Delta t^{m}}+\nabla \cdot\left\{\mathbf{u}_{w}^{k+1} c^{k, l, m+1}-D\left(s_{w}^{k+1}, \mathbf{u}_{w}^{k+1}, c_{s 1}^{k}, c_{s 2}^{k}\right)\right. \\
& \left.-R\left(\mathbf{u}_{w}^{k+1}, c_{s 1}^{k}\right)\right\} \nabla c^{k, l, m+1}=Q_{c}^{k, l, m+1},
\end{aligned}
$$

$$
\begin{gathered}
\frac{c_{s 1}^{k, l, m+1}-c_{s 1}^{k, l, m}}{\Delta t^{m}}= \\
\left\{\begin{array}{cc}
\gamma_{d}\left|\mathbf{u}_{w}^{k+1}\right| c^{k+1}, & \mathbf{u}_{w}^{k+1} \leq u_{r} \\
\gamma_{d}\left|\mathbf{u}_{w}^{k+1}\right| c^{k+1}-\gamma_{e}\left|\mathbf{u}_{w}^{k+1}-u_{r}\right| c_{s 1}^{k, l, m+1}, & \mathbf{u}_{w}^{k, l, m+1}>u_{r}
\end{array}\right.
\end{gathered}
$$

and,

$$
\frac{c_{s 2}^{k, l, m+1}-c_{s 2}^{k, l, m}}{\Delta t^{m}}=\gamma_{p t}\left|\mathbf{u}_{w}^{k+1}\right| c^{k, l, m+1}
$$

Finally, the permeability, porosity, and other parameters such as $\lambda_{w}, \lambda_{n}, \lambda_{t}$ and $f_{w}$ are updated. In the above system of equations both $\mathbf{K}$ and $\phi$ are functions in $c_{s 1}$ and $c_{s 2}$, that are functions of $u_{w}$ and $c$. On the other hand, $\mathbf{u}_{w}$ is a function of $\Phi_{w}$ and $s_{w}$, while $D$ is functions of $s_{w}, \mathbf{u}_{w}, c_{s 1}$ and $c_{s 2} . D$ and $R$ are functions of $s_{w}$ and $\mathbf{u}_{w}$. 


\section{Adaptive Time-Stepping}

In our algorithm, we have checked the Courant-Friedrichs-Lewy (CFL) condition to guarantee its satisfactory (i.e. $\mathrm{CFL}<1$ ). In order to achieve this idea, we have to define the following CFLs,

$$
\begin{aligned}
\mathrm{CFL}_{s, x} & =\frac{\mathbf{u}_{x} \Delta t^{k, l}}{\Delta x}, \\
\mathrm{CFL}_{s, y} & =\frac{\mathbf{u}_{y} \Delta t^{k, l}}{\Delta y},
\end{aligned}
$$

for saturation equation, and,

$$
\mathrm{CFL}_{c, x}=\frac{\mathbf{u}_{x} \Delta t^{k, l, m}}{\Delta x}
$$

and

$$
\mathrm{CFL}_{c, y}=\frac{\mathbf{u}_{y} \Delta t^{k, l, m}}{\Delta y}
$$

for concentration equations. It may be noted that the CFL depends on the ratio $\Delta t / \Delta x$ which can be fixed at larger time-steps and larger mesh-size. Thus, when we use a lager domain (larger mesh size) then we can use larger time step size. In the code, the initial time-step for the saturation equation is taken as the pressure time-step, i.e., $\Delta t^{k, 0}=\Delta t^{k}$, and the initial timestep for the concentration equation is taken as the saturation time-step, i.e., $\Delta t^{k, l, 0}=\Delta t^{k, l}$. Then, we check if $\mathrm{CFL}_{s, x}>1$ or $\mathrm{CFL}_{s, y}>1$, the saturation time-step will be divided by 2 and the $\mathrm{CFL}_{s, x}$ and $\mathrm{CFL}_{s, y}$ will be recalculated. 
This procedure will be repeated until satisfying the condition $\mathrm{CFL}_{s, x}<1$ and $\mathrm{CFL}_{s, y}<1$, then the final adaptive saturation time-step will be obtained. Similarly, we check if the condition, $\mathrm{CFL}_{c, x}>1$ or $\mathrm{CFL}_{c, y}>1$ is satisfied, the concentration time-step will be divided by 2 and therefore, we recalculate both $\mathrm{CFL}_{c, x}$ and $\mathrm{CFL}_{c, y}$. We repeat this procedure to reach the condition $\mathrm{CFL}_{c, x}<1$ and $\mathrm{CFL}_{c, y}<1$, then we obtain the final adaptive concentration time-step.

\section{Spatial Discretization}

The cell-centered finite difference (CCFD) is a locally conservative method that is very useful in solving petroleum reservoir simulation problems. Arbogast et al. [31] have proved that the CCFD method is equivalent to the mixed finite element method in the case of rectangular elements. In this work, we apply the CCFD scheme to the system of equations (16)-(22). The corresponding algebraic coupled pressure and saturation equation are solved implicitly to obtain the pressure is given as,

$$
\mathbf{A}_{t}\left(\mathbf{s}_{w}^{k}, \mathbf{c}_{s 1}^{k}, \mathbf{c}_{s 2}^{k}\right) \boldsymbol{\Phi}_{w}^{k+1}=\mathbf{Q}_{t}\left(\mathbf{s}_{w}^{k}, \mathbf{c}_{s 1}^{k}, \mathbf{c}_{s 2}^{k}\right)
$$

where

$$
\mathbf{A}_{t}\left(\mathbf{s}_{w}^{k}, \mathbf{c}_{s 1}^{k}, \mathbf{c}_{s 2}^{k}\right)=\mathbf{A}_{a}\left(\mathbf{s}_{w}^{k}, \mathbf{c}_{s 1}^{k}, \mathbf{c}_{s 2}^{k}\right)-\Delta t^{l} \mathbf{A}_{c}\left(\mathbf{s}_{w}^{k}, \mathbf{c}_{s 1}^{k}, \mathbf{c}_{s 2}^{k}\right) \boldsymbol{\Phi}^{\prime}\left(\mathbf{s}_{w}^{k}\right) \mathbf{M}^{-1} \mathbf{A}_{w}\left(\mathbf{s}_{w}^{k}, \mathbf{c}_{s 1}^{k}, \mathbf{c}_{s 2}^{k}\right),
$$


and,

$$
\begin{aligned}
\mathbf{Q}_{t}\left(\mathbf{s}_{w}^{k}, \mathbf{c}_{s 1}^{k}, \mathbf{c}_{s 2}^{k}\right)= & \mathbf{Q}_{a c}^{k+1}-\mathbf{A}_{c}\left(\mathbf{s}_{w}^{k}, \mathbf{c}_{s 1}^{k}, \mathbf{c}_{s 2}^{k}\right)\left[\boldsymbol{\Phi}_{c}\left(\mathbf{s}_{w}^{k}\right)+\boldsymbol{\Phi}^{\prime}\left(\mathbf{s}_{w}^{k}\right)\left(\mathbf{s}_{w}^{k}-\mathbf{s}_{w}^{k+1}\right)\right] \\
& -\Delta t^{l} \mathbf{A}_{c}\left(\mathbf{s}_{w}^{k}, \mathbf{c}_{s 1}^{k}, \mathbf{c}_{s 2}^{k}\right) \boldsymbol{\Phi}^{\prime}\left(\mathbf{s}_{w}^{k}\right) \mathbf{M}^{-1} \mathbf{Q}_{w}^{k+1}
\end{aligned}
$$

After calculating the pressure and the velocity, the CCFD scheme of (19) to update the saturation is the following algebraic equation,

$$
\mathbf{M}\left(\mathbf{c}_{s 1}^{k}, \mathbf{c}_{s 2}^{k}\right) \frac{\mathbf{s}_{w}^{k, l+1}-\mathbf{s}_{w}^{k}}{\Delta t^{l}}+\mathbf{A}_{s}\left(\mathbf{u}_{a}^{k+1}\right) f_{w}\left(\mathbf{s}_{w}^{k}\right)=\mathbf{Q}_{s}^{k, l+1}
$$

Similarly, the CCFD discretization of the equations of concentration (20)(22) can be obtained. The nanoparticles concentration CCFD equation is,

$$
\mathbf{B}_{t}\left(\mathbf{s}_{w}^{k+1}, \mathbf{u}_{w}^{k+1}, \mathbf{c}_{s 1}^{k}, \mathbf{c}_{s 2}^{k}\right) \mathbf{c}^{k, l, m+1}=\mathbf{Q}_{c t}\left(\mathbf{s}_{w}^{k}, \mathbf{c}^{k}, \mathbf{c}_{s 1}^{k}, \mathbf{c}_{s 2}^{k}\right)
$$

where

$$
\begin{array}{r}
\mathbf{B}_{t}\left(\mathbf{s}_{w}^{k+1}, \mathbf{u}_{w}^{k+1}, \mathbf{c}_{s 1}^{k}, \mathbf{c}_{s 2}^{k}\right)=\mathbf{B}_{m}\left(\mathbf{s}_{w}^{k+1}\right)+\Delta t^{m} \mathbf{M}\left(\mathbf{c}_{s 1}^{k}, \mathbf{c}_{s 2}^{k}\right)^{-1} \\
{\left[\mathbf{B}_{w}\left(\mathbf{u}_{w}^{k+1}\right)-\mathbf{B}_{D}\left(\mathbf{s}_{w}^{k+1}, \mathbf{u}_{w}^{k+1}, \mathbf{c}_{s 1}^{k}, \mathbf{c}_{s 2}^{k}\right)-\mathbf{R}\left(\mathbf{u}_{w}^{k+1}, \mathbf{c}_{s 1}^{k}\right)\right],}
\end{array}
$$

and,

$$
\mathbf{Q}_{c t}\left(\mathbf{s}_{w}^{k}, \mathbf{c}^{k}, \mathbf{c}_{s 1}^{k}, \mathbf{c}_{s 2}^{k}\right)=\Delta t^{m} \mathbf{M}\left(\mathbf{c}_{s 1}^{k}, \mathbf{c}_{s 2}^{k}\right)^{-1} \mathbf{Q}_{c}^{k, l, m+1}+\mathbf{B}_{m}\left(\mathbf{s}_{w}^{k}\right) \mathbf{c}^{k}
$$


The CCFD deposited nanoparticles concentration on the pore-walls is given by,

$$
\mathbf{c}_{s 1}^{k, l, m+1}=\left\{\begin{array}{cc}
\mathbf{R}_{1 t}\left(\mathbf{u}_{w}^{k+1}, \mathbf{c}^{k+1}, \mathbf{c}_{s 1}^{k}\right), & \mathbf{u}_{w}^{k+1} \leq u_{r} \\
{\left[\mathbf{I}+\Delta t^{m} \mathbf{R}_{2}\left(\mathbf{u}_{w}^{k+1}\right)\right]^{-1} \mathbf{R}_{1 t}\left(\mathbf{u}_{w}^{k+1}, \mathbf{c}^{k+1}, \mathbf{c}_{s 1}^{k}\right),} & \mathbf{u}_{w}^{k+1}>u_{r}
\end{array}\right.
$$

where

$$
\begin{gathered}
\mathbf{R}_{1 t}\left(\mathbf{u}_{w}^{k+1}, \mathbf{c}^{k+1}, \mathbf{c}_{s 1}^{k}\right)=\Delta t^{m} \mathbf{R}_{1}\left(\mathbf{u}_{w}^{k+1}, \mathbf{c}^{k+1}\right)+\mathbf{c}_{s 1}^{k}, \\
\mathbf{R}_{1}\left(\mathbf{u}_{w}^{k+1}, \mathbf{c}^{k+1}\right)=\gamma_{d}\left|\mathbf{u}_{w}\right| \mathbf{c}^{k+1}
\end{gathered}
$$

and,

$$
\mathbf{R}_{2}\left(\mathbf{u}_{w}^{k+1}\right)=\gamma_{e}\left|\mathbf{u}_{w}-u_{r}\right|
$$

Finally, one may write the CCFD discretization of the $\mathbf{c}_{s 2}$ equation as,

$$
\mathbf{c}_{s 2}^{k, l, m+1}=\Delta t^{m} \mathbf{R}_{3}\left(\mathbf{u}_{w}^{k+1}, \mathbf{c}^{k+1}\right)+\mathbf{c}_{s 2}^{k}
$$

where

$$
\mathbf{R}_{3}\left(\mathbf{u}_{w}^{k+1}, \mathbf{c}^{k+1}\right)=\gamma_{p t}\left|\mathbf{u}_{w}\right| \mathbf{c}^{k+1}
$$

We can solve the above equations to find, $\mathbf{s}^{k+1} \equiv \mathbf{s}^{k, l+1}, \mathbf{c}^{k+1} \equiv \mathbf{c}^{k, l, m+1}$, $\mathbf{c}_{s 1}^{k+1} \equiv \mathbf{c}_{s 1}^{k, l, m+1}$ and $\mathbf{c}_{s 2}^{k+1} \equiv \mathbf{c}_{s 2}^{k, l, m+1}$. 


\section{Numerical Tests}

In order to examine the performance of the current scheme, we introduce some numerical examples in this section. Firstly, we introduce the required physical parameters used in the computations. Then, we study the performance of the scheme by introducing some results for the adaptive time steps based on values of the corresponding Courant-Friedrichs-Lewy (CFL). Then we present some results for the distributions of water saturation and nanoparticles concentrations. In this study, given the normalized wetting phase saturation,

$$
S=\frac{s_{w}-s_{w r}}{1-s_{n r}-s_{w r}}, \quad 0 \leq S \leq 1
$$

the capillary pressure is defined as,

$$
p_{c}=-p_{e} \log S
$$

and the relative permeabilities are defined as,

$$
k_{r w}=k_{r w}^{0} S^{2}, \quad k_{r n}=k_{r n}^{0}(1-S)^{2},
$$

where

$$
k_{r w}^{0}=k_{r w}(S=1), k_{r w}^{0}=k_{r w}(S=1) .
$$

where $p_{e}$ is the capillary pressure parameter, $s_{w r}$ is the irreducible water saturation and $s_{n r}$ is the residual oil saturation after water flooding. The 
values and units of the physical parameters are inserted in Table 1.

Table 1: Values of the physical parameters .

\begin{tabular}{lll}
\hline Parameter & Value & Units \\
\hline$\gamma_{d}$ & 16 & $\mathrm{~m}^{-1}$ \\
$\gamma_{p t}$ & 1.28 & $\mathrm{~m}^{-1}$ \\
$\gamma_{e}$ & 30 & $\mathrm{~m}^{-1}$ \\
$\mathbf{u}_{r}$ & $4.6 \times 10^{-6}$ & $\mathrm{~m} / \mathrm{s}$ \\
$c_{0}$ & 0.1 & - \\
$S_{w r}$ & 0.001 & - \\
$S_{n r}$ & 0.001 & - \\
$\phi_{0}$ & 0.3 & - \\
$k_{f}$ & 0.6 & - \\
$\gamma_{f}$ & 0.01 & - \\
$\mu_{w}$ & 1 & $\mathrm{cP}=1.0 \times 10^{-3} \mathrm{~Pa} . \mathrm{s}$ \\
$\mu_{n}$ & 0.45 & $\mathrm{cP}$ \\
$k_{r w 0}$ & 1 & - \\
$k_{r o 0}$ & 1 & - \\
$B_{c}$ & 50 & $\mathrm{bar}=1.0 \times 10^{5} \mathrm{~Pa}$ \\
$D$ & $4.6 \times 10^{-8}$ & $\mathrm{~s}$ \\
\hline
\end{tabular}

In this example, we use a real permeability map of dimensions $120 \times 50$, which is extracted from Ref. [30]. The permeabilities vary in a large scope and are they highly heterogeneous as shown in Fig. 1. We consider a domain of size $40 \mathrm{~m} \times 16 \mathrm{~m}$ which is discretized into $120 \times 50$ uniform rectangles grids. The injection rate was 0.01 Pore-Volume-Injection (PVI) and continued the 


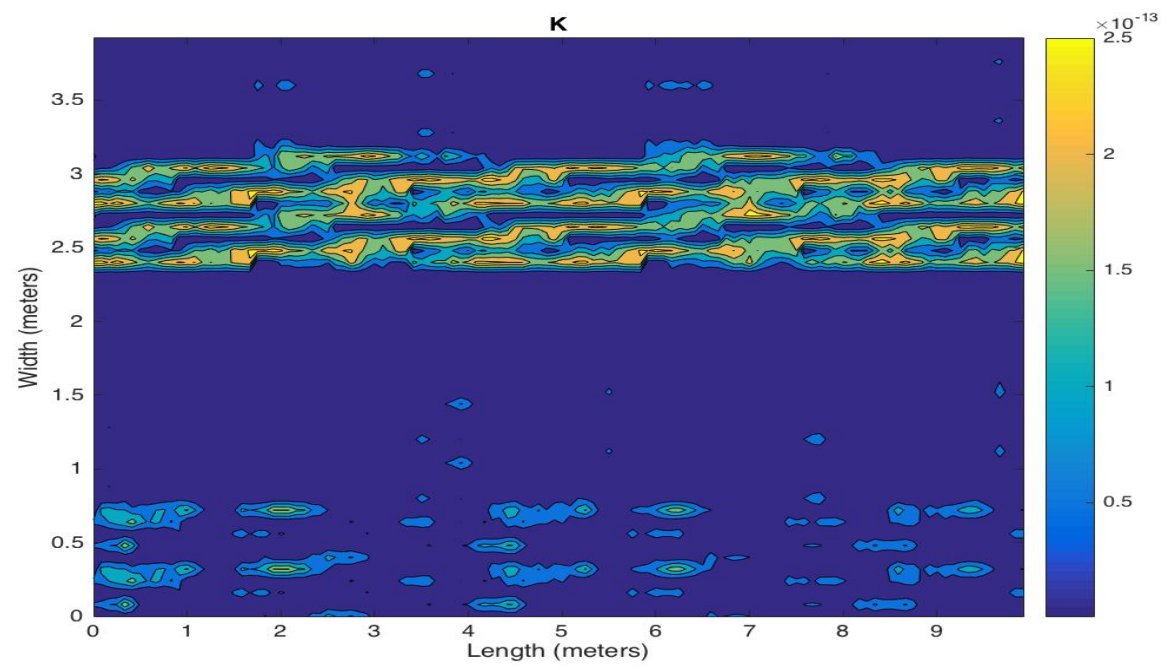

Figure 1: Real heterogenous permeability map.

calculation until 0.5 PV. In this example, we choose the number of steps of the outer loop to be: (Case 1, $k=50$, Fig. 2; Case 1, $k=100$, Fig. 2; Case 1, $k=200$, Fig. 3). In these figures, the adaptive time-step sizes, $\Delta t^{l}$ and $\Delta t^{m}$ are plotted against the number of steps of the outer loop $k$ and the number of the inner loops $l$ and $m$. It can be seen from these figures (Fig.2 - Fig. 4) that the behavior of adaptive $\Delta t^{l}$ and $\Delta t^{m}$ are very similar. This may be because the velocity is large and dominate the CFL. Also, both $\Delta t^{l}$ and $\Delta t^{m}$ start with large values then they gradually become smaller and smaller as $k$ increases. On the other hand, for the first two cases when $k=50,100$, $\Delta t^{l}$ and $\Delta t^{m}$ are small when $l$ and $m$ are small, then they increase to reach a peak, then they are gradually decreasing. However, in the third case when $k=200$, both $\Delta t^{l}$ and $\Delta t^{m}$ start with large values then they gradually become smaller and smaller as $l$ and $m$, respectively, increases.

Variations of saturation of the heterogenous permeability are shown in Fig. 5. It is noteworthy that the distribution for water saturation is dis- 

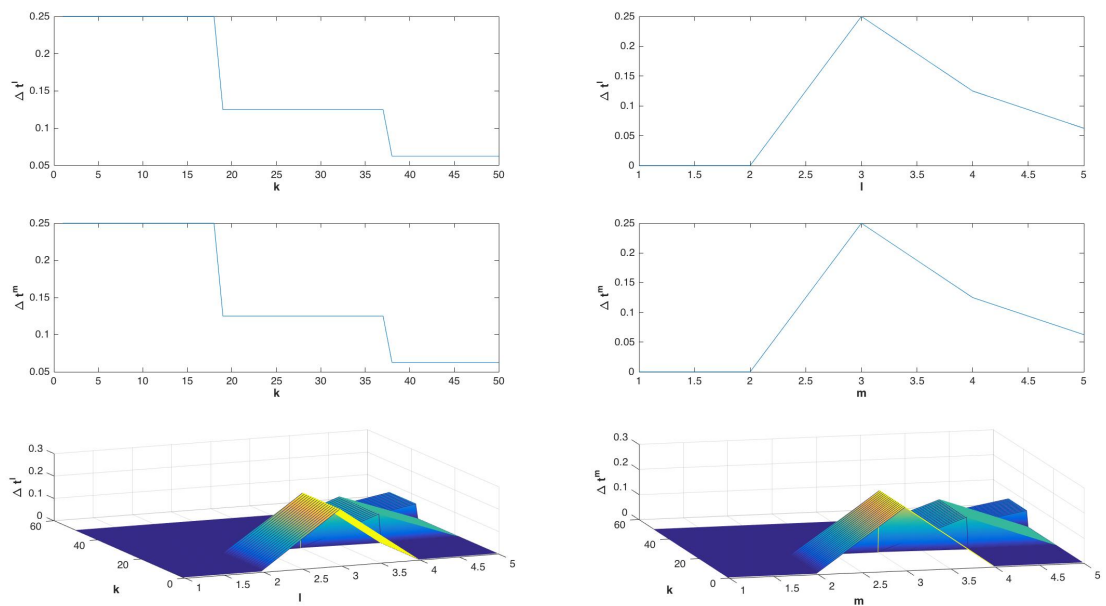

Figure 2: Adaptive time-step sizes, $\Delta t^{l}$ and $\Delta t^{m}$ against the number of steps of the outer loop $k$ and the number of the inner loops $l$ and $m$ : Case $1\left(\Delta t^{k}=50\right)$.
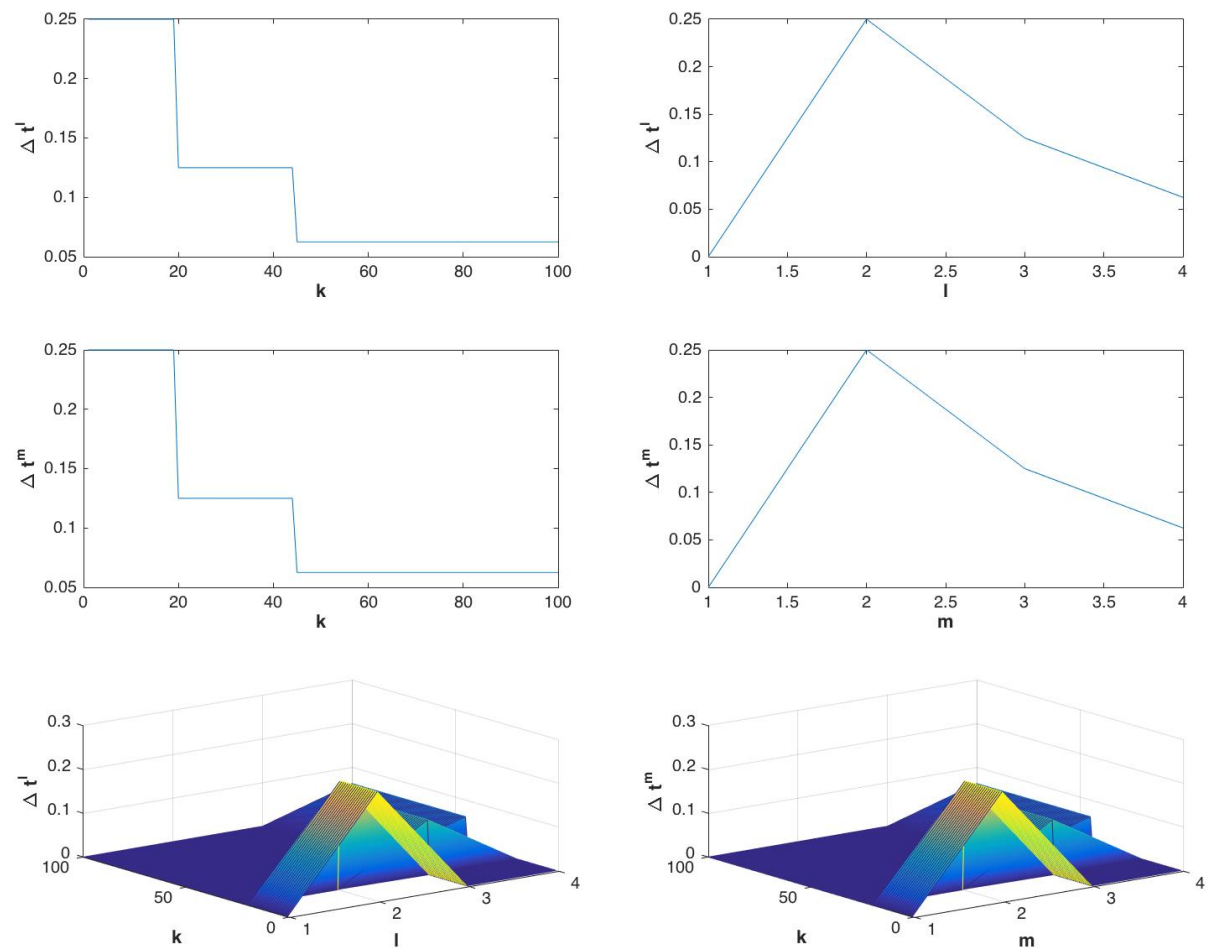

Figure 3: Adaptive time-step sizes, $\Delta t^{l}$ and $\Delta t^{m}$ against the number of steps of the outer loop $k$ and the number of the inner loops $l$ and $m$ : Case $2\left(\Delta t^{k}=100\right)$. 

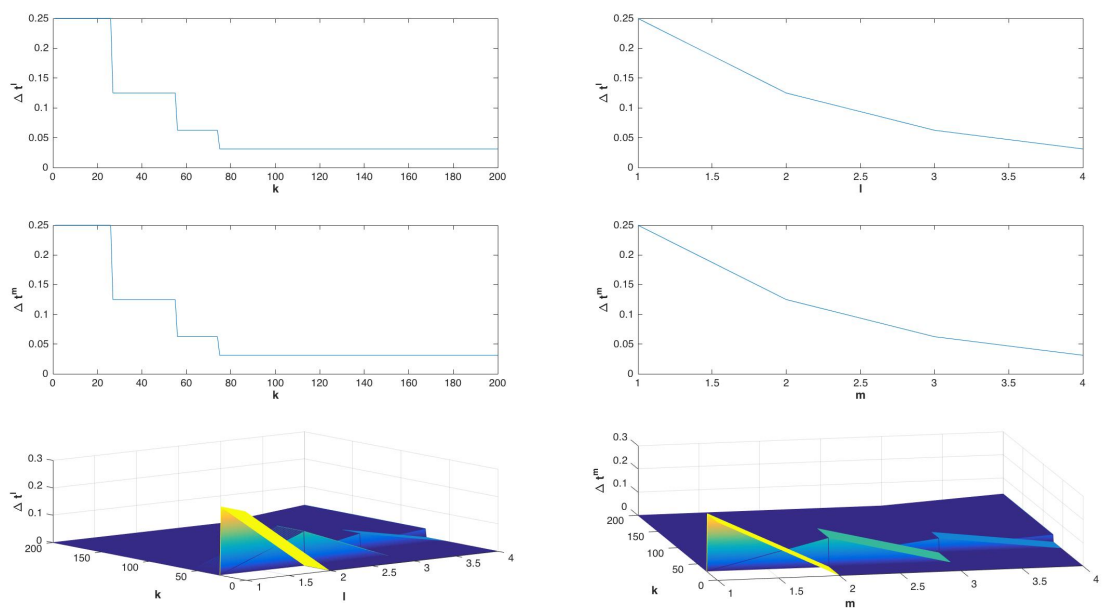

Figure 4: Adaptive time-step sizes, $\Delta t^{l}$ and $\Delta t^{m}$ against the number of steps of the outer loop $k$ and the number of the inner loops $l$ and $m$ : Case $3\left(\Delta t^{k}=200\right)$.

continuous due to the high heterogeneity. Thus, for example, we may note higher water saturation at higher permeability regions. Similarly, one may observe the discontinuity in the nanoparticles concentration as illustrated in Fig. 6. The contrast of the nanoparticles concentration distribution arisen from the heterogeneity of the medium can also be noted here. Moreover, the contours of the deposited nanoparticles concentration are shown in Fig. 7. The behavior of the entrapped nanoparticles concentration $c_{s 2}$ is very similar to the behavior of $c_{s 1}$ because of the similarity in their governing equation.

\section{Conclusions}

In this paper, we introduce an efficient time-stepping scheme with adaptive time-step sizes based on the CFL calculation. Hence, we have calculated the $\mathrm{CFL}_{s, x}, \mathrm{CFL}_{s, y}, \mathrm{CFL}_{c, x}$ and $\mathrm{CFL}_{c, y}$ at each substep and check if the 


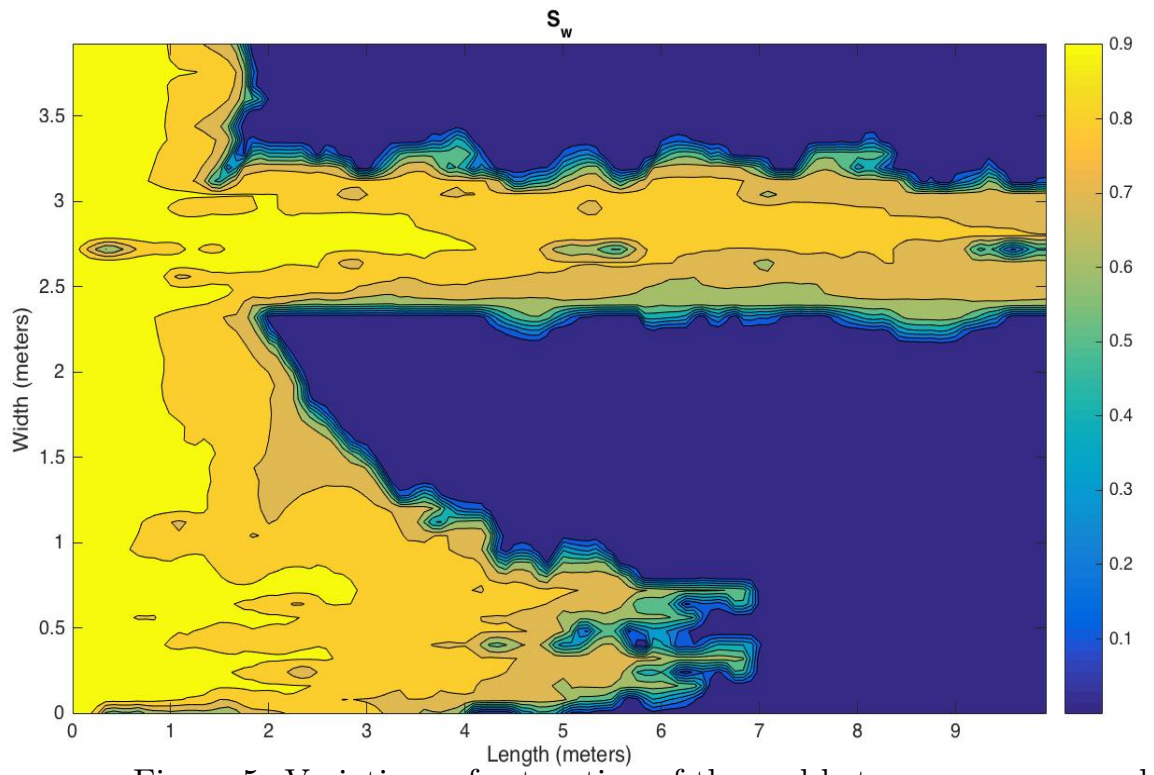

Figure 5: Variations of saturation of the real heterogenous permeability case.

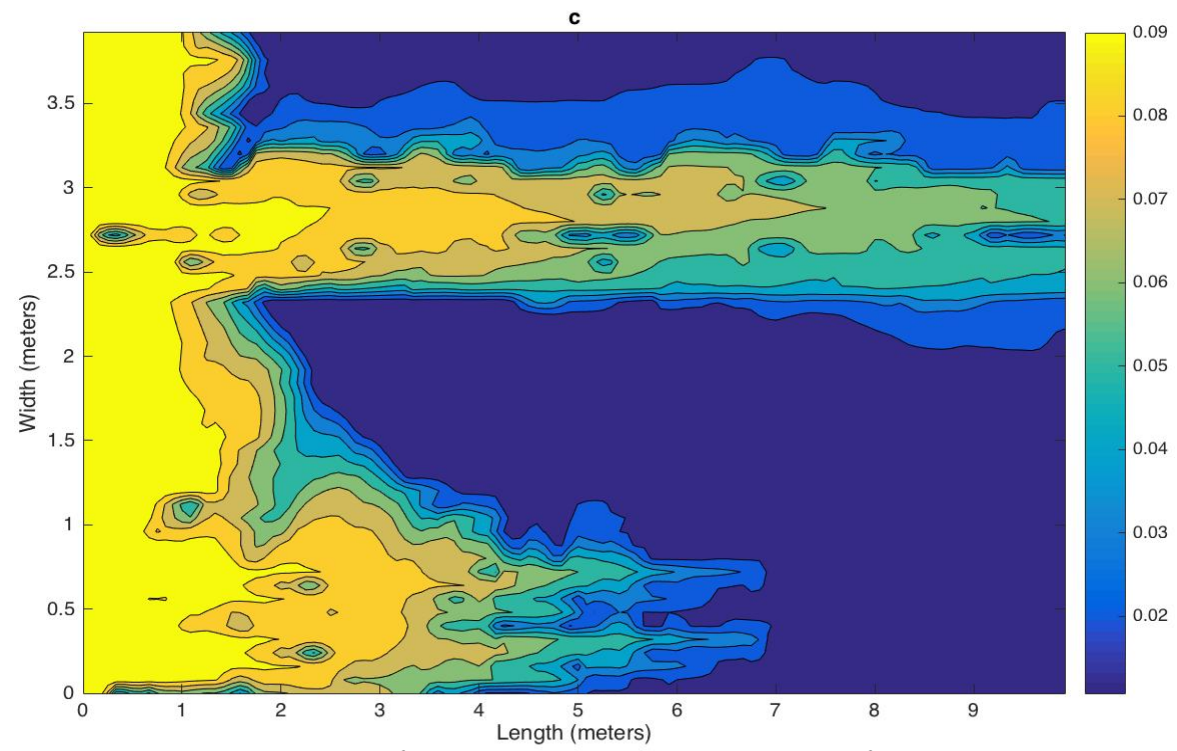

Figure 6: Variations of nanoparticles concentration of the real heterogenous permeability case. 


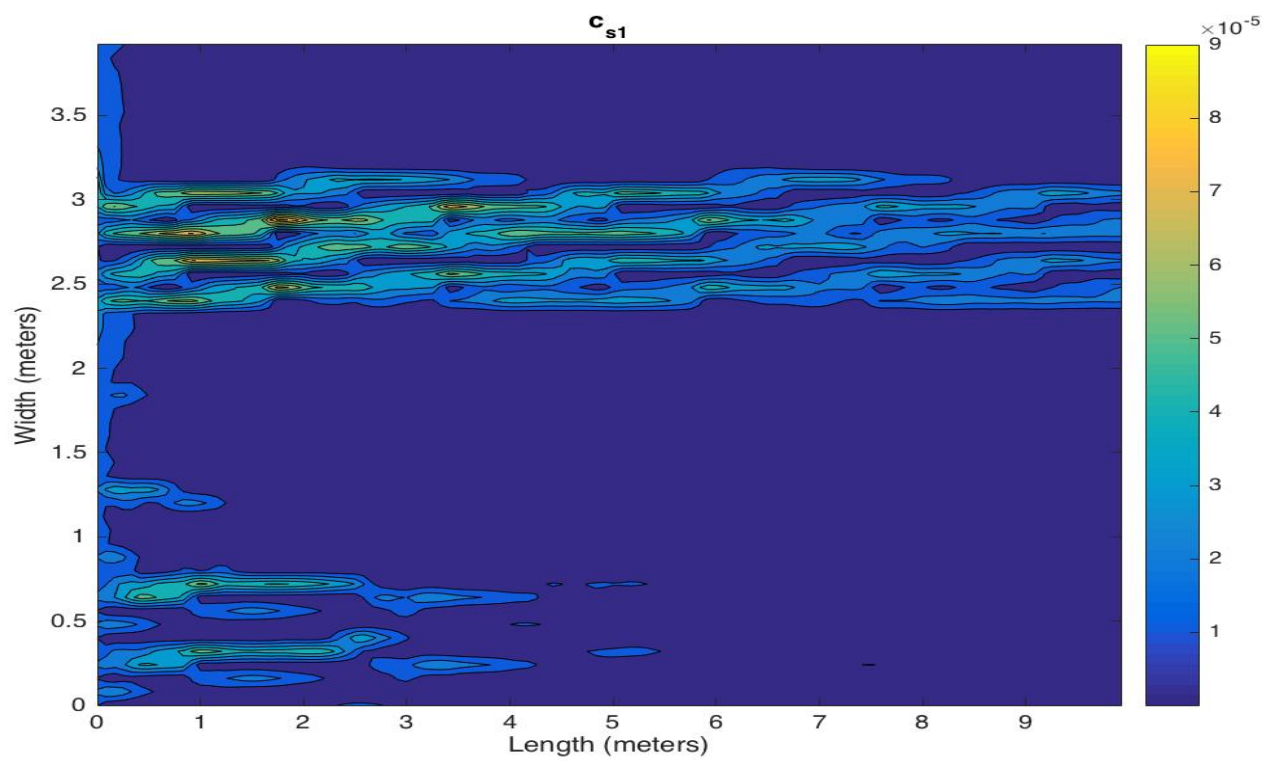

Figure 7: Variations of deposited nanoparticles concentration of the real heterogenous permeability case.

CFL condition is satisfied (i.e. $\mathrm{CFL}<1$ ). We applied this scheme with the IMES-IMC scheme to simulate the problem of nanoparticles transport in two-phase flow in porous media. The model consists of five differential equations, namely, pressure, saturation, nanoparticles concentration, deposited nanoparticles concentration on the pore-walls, and entrapped nanoparticles concentration in pore-throats. The capillary pressure is linearized and used to couple the pressure and the saturation equations. Then, the saturation equation is solved explicitly to update the saturation at each step. Therefore, the nanoparticles concentration equation is treated implicitly. Finally, the deposited nanoparticles concentration on the pore-walls equation, and entrapped nanoparticles concentration in pore-throats equation are solved implicitly. The CCFD method was used to discretize the governing equations spatially. In order to show the efficiency of the proposed scheme, we 
presented some numerical experiments. The outer pressure time-step size was selected then the inner ones, namely, the saturation subtime-step and the concentration subtime-step were calculated and adaptive by the CFL condition. We presented three cases with different values of the outer pressure time-step. Moreover, distributions of water saturation, nanoparticles concentration, and deposited nanoparticles concentration on pore-wall are shown in graphs.

\section{Acknowledgments}

The first author is thankful to the Effat University Deanship of Graduate

Studies and Researchfor providing the financial support through internal research grants system, Decision No. UC\#8/30.APR.2017/10.2-30(F).

\section{References}

[1] Coats, K.H. (2001), "IMPES stability: selection of stable time steps", SPE-84924, SPE ReservoirSimulation Symposium, Houston, TX.

[2] Chen, Z., Huan, G. and Ma, Y., "Computational methods for multiphase flows in porous media" SIAM Computational Science and Engineering, Philadelphia, 2006.

[3] Lu, Q. (2000), "A parallel multiblock/multiphysics approach for multiphase flow in porous media", Ph.D Thesis, The University of Texas at Austin. 
[4] Young, L.C. and Stephenson, R.E. (1983), "A generalized compositional approach for reservoir simulation", SPE Journal, Vol. 23, pp. 727-742.

[5] Kou, J. and Sun, S. (2010), "A new treatment of capillarity to improve the stability of IMPES two-phase flow formulation", Computers and Fluids, Vol. 39, pp. 1923-1931.

[6] T. Belytschko and Y. Y. Lu, "Convergence and stability analyses of multi-time step algorithm for parabolic systems," Computer Methods in Applied Mechanics and Engineering, vol. 102, no. 2, pp. 179-198, 1993.

[7] A. Gravouil and A. Combescure, "Multi-time-step explicit-implicit method for non-linear structural dynamics," International Journal for Numerical Methods in Engineering, vol. 50, no. 1, pp. 199-225, 2000.

[8] M. Klisinski, "Inconsistency errors of constant velocity multi-time step integration algorithms," Computer Assisted Mechanics and Engineering Sciences, vol. 8, no. 1, pp. 121-139, 2001.

[9] S. M. Bhallamudi, S. Panday, and P. S. Huyakorn, "Sub-timing in fluid flow and transport simulations," Advances in Water Resources, vol. 26, no. 5, pp. 477-489, 2003.

[10] Y. J. Park, E. A. Sudicky, S. Panday, J. F. Sykes, and V. Guvanasen, "Application of implicit sub-time stepping to simulate flow and trans- 
port in fractured porous media," Advances in Water Resources, vol. 31, no. 7, pp. 995-1003, 2008.

[11] V. Singh and S. M. Bhallamudi, "Complete hydrodynamic border-strip irrigation model," Journal of Irrigation and Drainage Engineering, vol. 122, no. 4, pp. 189-197, 1996.

[12] V. Singh and S. M. Bhallamudi, "Hydrodynamic modeling of basin irrigation," Journal of Irrigation and Drainage Engineering, vol. 123, no. 6, pp. 407-414, 1997.

[13] P. Smolinski, T. Belytschko, and M. Neal, "Multi-time-step integration using nodal partitioning," International Journal for Numerical Methods in Engineering, vol. 26, no. 2, pp. 349-359, 1988.

[14] P. Smolinski, S. Sleith, and T. Belytschko, "Stability of an explicit multitime step integration algorithm for linear structural dynamics equations," Computational Mechanics. Solids, Fluids, Engineered Materials, Aging Infrastructure, Molecular Dynamics, Heat Transfer, Manufacturing Processes, Optimization, Fracture \& Integrity, vol. 18, no. 3, pp. 236-243, 1996.

[15] S. Sun and J. Geiser, "Multiscale discontinuous Galerkin and operatorsplitting methods for modeling subsurface flow and transport," International Journal for Multiscale Computational Engineering, vol. 6, no. 1, pp. 87-101, 2008. 
[16] J. E. VanderKwaak, Numerical simulation of flow and chemical transport in integrated surface-subsurface hydrologic systems, Ph.D. thesis, University of Waterloo, 1999.

[17] Hoteit, H. and Firoozabadi, A. (2008), "Numerical modeling of twophase flow in heterogeneous permeable media with different capillarity pressures", Advances in Water Resources, Vol. 31, pp. 56-73.

[18] Gruesbeck, C. and Collins, R.E. (1982), "Entrainment and deposition of fines particles in porous media", SPE Journal, Vol. 24, pp. 847-856.

[19] Ju, B. and Fan, T. (2009), "Experimental study and mathematical model of nanoparticle transport in porous media", Powder Technology, Vol.192, pp. 195-202.

[20] Ju, B., Fan, T. and Qiu, X. (2002), "A study of wettability and permeability change caused by adsorption of nanometer structured polysilicon on the surface of porous media", SPE-77938, SPE Asia Pacific Oil and Gas Conference and Exhibition, Melbourne, Australia.

[21] Liu, X.H. and Civian, F. (1994), "Formation damage and skin factor due to filter cake formation and fines migration in the Near-Wellbore Region", SPE-27364, SPE Symposium on Formation Damage Control, Lafayette, Louisiana.

[22] El-Amin, M.F., Salama, A. and Sun, S. (2012), "Modeling and simulation of nanoparticles transport in a two-phase flow in porous media", 
SPE-154972, SPE International Oilfield Nanotechnology Conference and Exhibition, Noordwijk, The Netherlands.

[23] El-Amin, M.F., Sun, S. and Salama, A. (2012), "Modeling and simulation of nanoparticle transport in multiphase flows in porous media: $\mathrm{CO}_{2}$ sequestration" SPE-163089, Mathematical Methods in Fluid Dynamics and Simulation of Giant Oil and Gas Reservoirs.

[24] El-Amin, M.F., Sun, S. and Salama, A. (2013), "Enhanced oil recovery by nanoparticles injection: modeling and simulation" SPE-164333, SPE Middle East Oil and Gas Show and Exhibition held in Manama, Bahrain.

[25] El-Amin, M.F., Salama, A. and Sun, S. (2015), "Numerical and dimensional analysis of nanoparticles transport with two-phase flow in porous media", Journal of Petroleum Science and Engineering, Vol. 128, pp. 53-64.

[26] Salama, A., Negara, A., El-Amin, M.F. and Sun, S. (2015), "Numerical investigation of nanoparticles transport in anisotropic porous media" Journal of Contaminant Hydrology, Vol. 181, pp. 114-130.

[27] H. Chen, Q. Di, F. Ye, C. Gu and J. Zhang, "Numerical simulation of drag reduction effects by hydrophobic nanoparticles adsorption method in water flooding processes," Journal of Natural Gas Science and Engineering, Vol. 35, pp. 1261-1269, 2016. 
[28] Chen, M.H., Salama, A. and El-Amin, M.F. (2016), "Numerical aspects related to the dynamic update of anisotropic permeability field during the transport of nanoparticles in the subsurface", Procedia Computer Science, Vol. 80, pp. 1382-1391.

[29] El-Amin, M.F., Kou, J., Salama, A. and Sun, S. (2016), "An iterative implicit scheme for nanoparticles transport with two-Phase flow in porous media" Procedia Computer Science, Vol. 80, pp. 1344-1353.

[30] Al-Dhafeeri, A.M. and Nasr-El-Din, H.A. (2007), "Characteristics of high-permeability zones using core analysis, and production logging data", Journal of Petroleum Science and Engineering, Vol. 55, pp. 1836.

[31] Arbogast, T. and Wheeler, M.F. and Yotov, I (1997), "Mixed finite elements for elliptic problems with tensor coefficients as cell-centered finite differences", SIAM Journal on Numerical Analysis, Vol. 34, no. 2, pp. $828-852$. 